\title{
Nuclear obscuration and scattering in Seyfert 2 galaxies
}

\author{
Q. Gu ${ }^{1,2}$, R. Maiolino ${ }^{3}$, and D. Dultzin-Hacyan ${ }^{2}$ \\ 1 Department of Astronomy, Nanjing University, Nanjing 210093, PR China \\ 2 Instituto de Astronomía, Universidad Nacional Autónoma de Mexico, Apdo Postal 70-264, Mexico DF 04510, \\ Mexico \\ ${ }^{3}$ Osservatorio Astrofisico di Arcetri, Largo Enrico Fermi 5, 5015 Firenze, Italy
}

Received 19 October 2000 / Accepted 13 November 2000

\begin{abstract}
We study the relationship between gaseous absorbing column density $\left(N_{\mathrm{H}}\right)$, infrared colors and detectability of the broad lines in a large sample of Seyfert 2 galaxies(Sy2s). We confirm that Sy2s without polarized broad lines tend to have cooler $60 \mu \mathrm{m} / 25 \mu \mathrm{m}$ colors; this correlation was previously ascribed to the effect of obscuration towards the nuclear region. We find some evidence that Sy2s without polarized broad lines have larger absorbing column density $\left(N_{\mathrm{H}}\right)$ and that a fraction of them are characterized by dust lanes crossing their nuclei. However, we find that the IR colors do not correlate with $N_{\mathrm{H}}$, in disagreement with the obscuration scenario. Also, Sy2s without polarized broad lines follow the same radio-FIR relation as normal and starburst galaxies, at variance with Sy2s with polarized broad lines. These results indicate that the lack of broad lines in the polarized spectrum of Sy2s is mostly due to the contribution/dilution from the host galaxy or from a circumnuclear starburst, though a lesser extent to the obscuration toward the nuclear region also plays a role.
\end{abstract}

Key words. galaxies: active - galaxies: ISM - galaxies: Seyfert - galaxies: statistics

\section{Introduction}

According to the standard unification model, Seyfert 1 and 2 galaxies (Sy1s and Sy2s hereafter) are intrinsically the same objects and the absence of broad lines in Sy2s is ascribed to the obscuration by a pc-scale dusty torus oriented along the line of sight (see the reviews by Antonucci 1993; Véron-Cetty \& Véron 2000a). The observational evidence for this model includes the detection of polarized broad emission lines in some Seyfert 2 galaxies (Antonucci \& Miller 1985; Tran 1995; Moran et al. 2000), the detection of broad lines in the infrared spectrum of some Sy2s (Ruiz et al. 1994; Veilleux et al. 1997; Rix et al. 1990) and the detection of a prominent photoelectric cutoff in the X-ray spectra of Sy2s indicating the presence of large columns of gas along the line of sight (Koyama et al. 1989; Awaki et al. 1991; Maiolino et al. 1998; Risaliti et al. 1999).

The spectropolarimetric observations of different samples of Seyfert 2 galaxies indicate that only about $40 \%$ of Sy2s show broad lines in their polarized spectra (e.g. Heisler et al. 1997), although such surveys are probably biased, since pre-selection was done according to broadband polarization.

According to the suggestion of Heisler et al. (1997), the detectability of a hidden BLR through spectropolarimetry in Sy2s is related to the inclination of the torus which,

Send offprint requests to: Q. Gu, e-mail: qsgu@nju.edu.cn in turn, is related to the $60 \mu \mathrm{m}$ to $25 \mu \mathrm{m}$ flux ratio, $s_{60 \mu \mathrm{m}} / s_{25 \mu \mathrm{m}}$. More specifically, in those Sy2s showing polarized broad lines (PBL), the torus should be oriented more face-on so that the scattering medium is less obscured by the torus itself. This model would also explain the correlation between IR colors $\left(s_{60 \mu \mathrm{m}} / s_{25} \mu \mathrm{m}\right)$ and detectability of the PBL. In particular, when the torus is observed close to pole-on, the PBL should be easier to detect and the IR color should be hotter, since we are observing the hotter dust-emitting region, in agreement with observations.

However, more recently, Alexander (2000) compared the absorbing column densities inferred from hard X-rays with the detectability of the PBL and found no correlation. This result is in contrast with the Heisler et al. (1997) model, which would predict a higher absorbing column for Sy2s without PBL. Alexander (2000) suggests that the relation between detectability of the PBL and IR colors is indirect: the contribution from the host galaxy would both make the IR color cooler and would also dilute the nuclear optical spectrum, making more difficult the detection of scattered polarized light.

The simple model of the obscuring torus has been subject to various modifications. In particular, while the pcscale torus is probably responsible for the huge absorbing columns $\left(N_{\mathrm{H}}>10^{24} \mathrm{~cm}^{-2}\right)$ observed in several Sy2s, observational evidence has also been found for a larger scale 
( $\sim 100$ pc) obscuring medium with a lower absorbing column density $\left(N_{\mathrm{H}} \sim\right.$ a few $\times 10^{22} \mathrm{~cm}^{-2}$, Granato et al. 1997; Matt 2000; Maiolino 2000 and references therein).

In this paper we expand the work done by Alexander (2000) by enlarging the sample of Sy2s for which information on both the absorbing $N_{\mathrm{H}}$ and on the detection of PBL is available. We also seek additional constraints on the nature of the circumnuclear scattering and absorbing medium by comparing the mid- and far-IR colors with $N_{\mathrm{H}}$ and the detectability of PBL with the nuclear morphology and with the radio power.

The paper is organized as follows. In Sect. 2 we present our sample of Seyfert 2 galaxies with spectropolarimetric observations; the results are given in Sect. 3. We discuss our results and their implications in Sect. 4 and summarize our conclusions in Sect. 5 .

\section{The sample}

We collected all Seyfert 2 galaxies from the recent literature (from 1985 to 2000), for which both spectropolarimetric data and an estimate of $N_{\mathrm{H}}$ from X-rays are available. Within this sample, we report 22 Seyfert 2 galaxies with PBL, and 18 Sy2s without detection of PBL, which are presented in Tables 1 and 2, respectively.

In Tables 1 and 2, we report the following information: galaxy name (Col. 1$)$; column density $\left(N_{\mathrm{H}}\right)$ taken from Bassani et al. (1999); Risaliti et al. (1999); and Alexander (2000) (Col. 2); the IRAS colors $s_{25} \mu \mathrm{m} / s_{12 \mu \mathrm{m}}$ and $s_{60 \mu \mathrm{m}} / s_{25} \mu \mathrm{m}$ in Cols. 3 and 4 , where the IRAS fluxes are taken from Moshir et al. (1992); and the flux between 42.5 and $122.5 \mu \mathrm{m}, \mathrm{FIR}$, where FIR $=1.2610^{-14}(2.58 \times$ $\left.s_{60 \mu \mathrm{m}}+s_{100 \mu \mathrm{m}}\right)$, in Col. 5 ; the $1.49 \mathrm{GHz}$ radio emission from the NRAO/VLA Sky Survey (NVSS) (Condon et al. 1998) in Col. 6; and the corresponding reference for PBL in Col. 7.

\section{The results}

\section{1. $N_{\mathrm{H}}$ versus detectability of polarized broad lines}

We show the histogram distribution of column densities for Sy2s without and with PBL in Figs. 1a and 1b, respectively. Since there are 6 censored data (lower limits) among Sy2s with PBL and 11 among Sy2s without PBL, we need to use the survival analysis methods (ASURV Rev 1.2, Isobe, Feigelson \& Nelson 1986) to study the similarity between these two samples. We find that the probability for these two samples to be extracted from the same parent population is about $12 \%$, and the mean values of $\log N_{\mathrm{H}}$ (in units of $\mathrm{cm}^{-2}$ ) are $23.6 \pm 0.2$ and $24.2 \pm$ 0.2 , respectively. This result suggests that Sy2s with PBL are affected by lower obscuration than Sy2s without PBL, but the statistical significance of the result is not high, and therefore not conclusive.

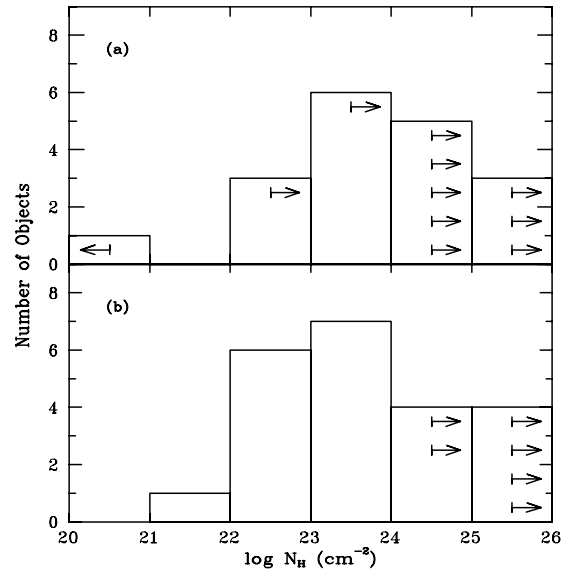

Fig. 1. Distribution of the absorbing column densities $\left(N_{\mathrm{H}}\right)$ for Seyfert 2 galaxies without polarized broad lines (Fig. 1a) and for Sy2s with detected polarized broad lines (Fig. 1b)

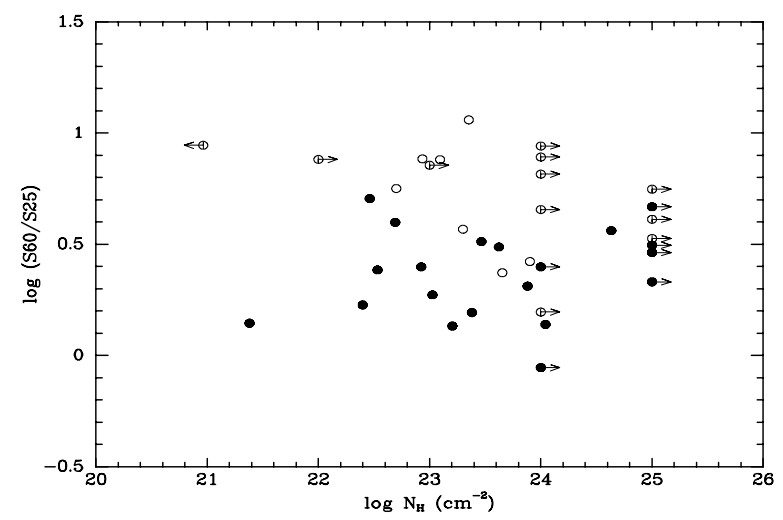

Fig. 2. Distribution of the absorbing column density $\left(N_{\mathrm{H}}\right)$ versus the IR color $\left(s_{60} \mu \mathrm{m} / s_{25} \mu \mathrm{m}\right)$. Seyfert 2 galaxies with and without PBL are marked with filled and open circles, respectively

\subsection{Infrared colors versus $N_{H}$}

In Fig. 2, we show the plot of column density $\left(N_{\mathrm{H}}\right)$ versus far-IR color $\left(s_{60} \mu \mathrm{m} / s_{25} \mu \mathrm{m}\right)$ for Seyfert 2 galaxies with PBL (filled circles) and Sy2s without PBL (open circles). Sy2s with PBL have warmer FIR colors than Sy2s without PBL, which confirms the result obtained by Heisler et al. (1997) with a higher statistical significance; the probability that these two samples originate from the same parent population is less than $0.0001 \%$ and the mean values of $\log \left(s_{60 \mu \mathrm{m}} / s_{25} \mu \mathrm{m}\right)$ are $0.368 \pm 0.044$ and $0.722 \pm 0.053$, respectively. However, we do not find any correlation between the $s_{60 \mu \mathrm{m}} / s_{25} \mu \mathrm{m}$ color and the absorbing $N_{\mathrm{H}}$, at variance with what is expected from the model proposed by Heisler et al. (1997): according to the latter model, higher $N_{\mathrm{H}}$ should correspond to cooler IR colors.

Figure 3 is a plot similar to Fig. 2, but where the IR color is sampled at shorter wavelengths and, more specifically, the ratio $s_{60 \mu \mathrm{m}} / s_{25} \mu \mathrm{m}$ is replaced by $s_{25} \mu \mathrm{m} / s_{12} \mu \mathrm{m}$. This ratio should be more sensitive to absorption of the inner (hotter) dust component. In this case, there is marginal evidence that Sy2s with $N_{\mathrm{H}}<10^{23} \mathrm{~cm}^{-2}$ have 
Table 1. Basic data for Seyfert 2 galaxies with PBL

\begin{tabular}{|c|c|c|c|c|c|c|c|}
\hline Name & & $N_{\mathrm{H}}{ }^{\mathrm{a}}$ & $s_{25 \mu \mathrm{m}} / s_{12 \mu \mathrm{m}}$ & $s_{60 \mu \mathrm{m}} / s_{25 \mu \mathrm{m}}$ & FIR $^{\mathrm{b}}$ & $F_{1.49 \mathrm{GHz}}{ }^{\mathrm{c}}$ & Ref \\
\hline Circinus & & $43000_{-11000}^{+19000}$ & 3.640 & 3.634 & 12.065 & & $\mathrm{~h}$ \\
\hline ESO $434-$ G40 & & $162_{-21}^{+23}$ & & & & 14.6 & $\mathrm{~h}$ \\
\hline F05189-2524 & & $490_{-16}^{+10}$ & 4.632 & 3.960 & 0.600 & 29.1 & $\mathrm{~h}$ \\
\hline F09104+4109 & & $24_{-6}^{+6}$ & 1.520 & 1.395 & 0.030 & & $\mathrm{j}$ \\
\hline F13197-1627 & & 7943 & 3.135 & 2.047 & 0.259 & 275.3 & $\mathrm{~d}$ \\
\hline F20460+1925 & & $250_{-32}^{+34}$ & 1.385 & 1.685 & 0.042 & 18.9 & $\mathrm{~h}$ \\
\hline $\mathrm{F} 23060+0505$ & & $840_{-250}^{+190}$ & 1.438 & 2.500 & 0.051 & 6.8 & $\mathrm{~h}$ \\
\hline IC 3639 & $>$ & 100000 & 3.538 & 3.130 & 0.374 & & $\mathrm{i}$ \\
\hline IC 5063 & & $2400_{-200}^{+200}$ & 3.310 & 1.557 & 0.250 & & $\mathrm{k}$ \\
\hline Mark 3 & & $11000_{-2500}^{+1500}$ & 4.057 & 1.377 & 0.171 & 1100.9 & e \\
\hline Mark 348 & & $1060_{-260}^{+310}$ & 2.484 & 1.870 & 0.070 & 292.7 & e \\
\hline Mark 463E & & $1600_{-800}^{+800}$ & 2.825 & 1.354 & 0.094 & 381.0 & e \\
\hline Mark 477 & $>$ & 10000 & 2.160 & 2.500 & 0.067 & 60.8 & $\mathrm{f}$ \\
\hline Mark 1210 & $>$ & 10000 & 3.800 & 0.880 & 0.079 & 114.9 & $\mathrm{f}$ \\
\hline NGC 1068 & $>$ & 100000 & 2.267 & 2.140 & 9.070 & 4849.0 & $\mathrm{~g}$ \\
\hline NGC 2110 & & $289_{-29}^{+21}$ & 2.378 & 5.068 & 0.224 & 299.4 & $\mathrm{~h}$ \\
\hline NGC 2273 & $>$ & 100000 & 2.957 & 4.654 & 0.335 & 63.4 & $\mathrm{i}$ \\
\hline NGC 3081 & & $6600_{-1600}^{+1800}$ & & & & 5.7 & o \\
\hline NGC 4388 & & $4200_{-1000}^{+600}$ & 3.550 & 3.070 & 0.578 & 120.4 & $\mathrm{~h}$ \\
\hline NGC 4507 & & $2920_{-230}^{+230}$ & 3.065 & 3.248 & 0.219 & 67.4 & o \\
\hline NGC 5506 & & $340_{-12}^{+26}$ & 2.800 & 2.420 & 0.409 & 339.4 & $\mathrm{~h}$ \\
\hline NGC 7674 & $>$ & 100000 & 2.667 & 2.901 & 0.288 & 221.4 & $\mathrm{e}$ \\
\hline
\end{tabular}

Table 2. Basic data for Seyfert 2 galaxies without PBL

\begin{tabular}{|c|c|c|c|c|c|c|c|}
\hline Name & & $\overline{N_{\mathrm{H}}{ }^{\mathrm{a}}}$ & 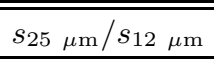 & 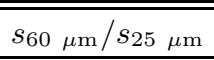 & $\mathrm{FIR}^{\mathrm{b}}$ & $F_{1.49 \mathrm{GHz}^{\mathrm{c}}}$ & Ref \\
\hline F19254-7245 & & $1995^{\mathrm{d}}$ & 5.462 & 3.690 & 0.249 & & 1 \\
\hline Mark 1066 & $>$ & 10000 & 4.620 & 4.524 & 0.505 & & $\mathrm{o}$ \\
\hline NGC 34 & $>$ & $1000^{\mathrm{d}}$ & 5.667 & 7.155 & 0.779 & 67.5 & 1 \\
\hline NGC 1143 & $>$ & $100^{\mathrm{d}}$ & 2.692 & 7.600 & 0.319 & & 1 \\
\hline NGC 1386 & $>$ & 100000 & 2.880 & 4.090 & 0.316 & 37.8 & o \\
\hline NGC 1667 & $>$ & 10000 & 1.763 & 8.731 & 0.375 & 77.3 & $\mathrm{o}$ \\
\hline NGC 3281 & & $7980_{-1500}^{+1900}$ & 2.909 & 2.641 & 0.317 & 80.9 & o \\
\hline NGC 3393 & $>$ & 100000 & 2.840 & 3.352 & 0.127 & 81.5 & $\mathrm{~m}$ \\
\hline NGC 4941 & & $4500_{-1400}^{+2500}$ & 2.280 & 2.351 & 0.095 & 20.3 & o \\
\hline NGC 5128 & & $2250_{-1250}^{+1250}$ & 1.346 & 11.441 & 9.829 & & $\mathrm{n}$ \\
\hline NGC 5135 & $>$ & 10000 & 3.701 & 6.524 & 0.914 & 1.5 & 1 \\
\hline NGC 5347 & $>$ & 10000 & 3.172 & 1.565 & 0.081 & & o \\
\hline NGC 5643 & $>$ & 100000 & 3.895 & 5.585 & 1.165 & & o \\
\hline NGC 7130 & $>$ & 10000 & 3.397 & 7.790 & 0.873 & 190.6 & 1 \\
\hline NGC 7172 & & $861_{-33}^{+79}$ & 1.696 & 7.641 & 0.356 & 37.6 & 1 \\
\hline NGC 7496 & & $501^{\mathrm{d}}$ & 5.630 & 5.625 & 0.471 & & 1 \\
\hline NGC 7582 & & $1240_{-80}^{+60}$ & 4.689 & 7.585 & 2.477 & & 1 \\
\hline NGC 7590 & $<$ & 9.2 & 1.615 & 8.798 & 0.467 & & 1 \\
\hline
\end{tabular}

${ }^{\mathrm{a}}$ Absorbing column density in units of $10^{20} \mathrm{~cm}^{-2} ;{ }^{\mathrm{b}}$ In units of $10^{-12} \mathrm{~W} \mathrm{~m}^{-2} ;{ }^{\mathrm{c}}$ In units of mJy; ${ }^{\mathrm{d}}$ Alexander $(2000) ;{ }^{\mathrm{e}}$ Miller \& Goodrich (1990); ${ }^{\mathrm{f}}$ Tran et al. (1992); ${ }^{\mathrm{g}}$ Antonucci \& Miller (1985); ${ }^{\mathrm{h}}$ Véron-Cetty \& Véron (2000b); ${ }^{\mathrm{i}}$ Kay $(2000) ;{ }^{\mathrm{j}}$ Hines \& Wills (1993); ${ }^{\mathrm{k}}$ Inglis et al. (1993); ${ }^{1}$ Heisler et al. (1997); ${ }^{\mathrm{m}}$ Nagao et al. (2000); ${ }^{\mathrm{n}}$ Alexander et al. (1999); ${ }^{\circ}$ Moran et al. (2000). 


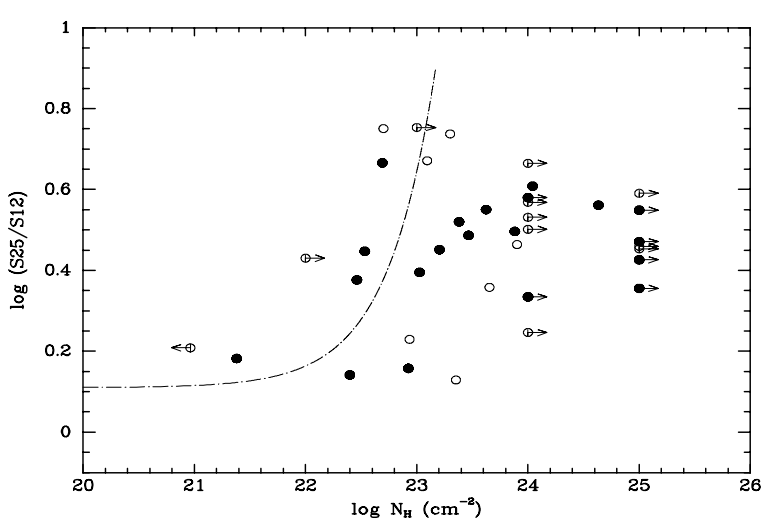

Fig. 3. Distribution of the absorbing column density $\left(N_{\mathrm{H}}\right)$ versus the IR color $\left(s_{25} \mu \mathrm{m} / s_{12} \mu \mathrm{m}\right)$. Symbols have the same coding as in Fig. 2. The dot-dashed line indicates the expected relation between IR color and absorbing $N_{\mathrm{H}}$, assuming that the intrinsic IR color is the same as observed in Sy1s and that the dust associated with the observed $N_{\mathrm{H}}$ completely covers the IR-emitting region

warmer colors. Yet, the relation between $s_{25} \mu \mathrm{m} / s_{12} \mu \mathrm{m}$ color and $N_{\mathrm{H}}$ is not the one expected from the absorption by a dusty screen associated with the gaseous column observed in the X-ray, assuming a Galactic gas-to-dust ratio and extinction curve. Such a relation is given by the following formula:

$\log \left(\frac{s_{25}}{s_{12}}\right)_{\mathrm{obs}}=\log \left(\frac{s_{25}}{s_{12}}\right)_{\mathrm{int}}+0.042 \times N_{\mathrm{H}} 10^{-22}$

where $\left(s_{25} \mu \mathrm{m} / s_{12} \mu \mathrm{m}\right)$ int is the intrinsic color prior to absorption and where we assumed

$A_{12}=\frac{1}{27} A_{V} \quad$ and $\quad A_{25}=\frac{1}{55} A_{V}$

and

$A_{v}=5.010^{-22} N_{\mathrm{H}}$

(Bohlin et al. 1978). To estimate the intrinsic $s_{25 \mu \mathrm{m}} / s_{12 \mu \mathrm{m}}$ color, we selected all of the Seyfert 1 galaxies listed in the Véron-Cetty \& Véron (2000b) catalog with available IRAS fluxes and we derived a mean value of $\left\langle\log \left(\frac{s_{25}}{s_{12}}\right)\right\rangle=0.11$. Therefore, within the framework of the unified model, we assigned this value to $\log \left(\frac{s_{25}}{s_{12}}\right)_{\text {int }}$ in Eq. (1). The resulting curve is shown in Fig. 3 with a dot-dashed line, which may match the observational data for low $N_{\mathrm{H}}\left(<10^{23} \mathrm{~cm}^{-2}\right)$, but fails to account for the majority of the sources at higher columns. This finding indicates that the observed distribution of IR colors cannot be ascribed to different degrees of absorption (at least for $N_{\mathrm{H}}>10^{23} \mathrm{~cm}^{-2}$ ).

\subsection{Detectability of polarized broad lines versus nuclear morphology}

HST images of a large sample of Seyfert galaxies have shown that Seyfert 2 galaxies are characterized by dust lanes or irregular dust distributions crossing the nuclear region more often than Sy1s. Based on these results, Malkan et al. (1998) suggested that 100 pc-scale dusty structures may play a role in the obscuration that generally affects Sy2s. As summarized in Maiolino (2000) the presence of a 100 pc-scale obscuring medium in Sy2s is supported by various pieces of evidence, but most likely such a large scale medium contributes to the absorption only with "moderate" gas columns (less than a few $\left.\times 10^{23} \mathrm{~cm}^{-2}\right)$.

Among all of the Seyfert 2 galaxies imaged by Malkan et al. (1998), we searched those observed in spectropolarimetry and found 32. Out of these 32 Sy2s, 12 have PBL and 20 do not have PBL. $30 \%$ of the Sy2s without PBL are characterized by (large scale) dust lanes crossing the nuclear region or irregular nuclear dust distribution (according to the classification given in Malkan et al. 1998), while none of the Sy2s with PBL show evidence for dusty nuclear features. This suggests that, at least in some cases, obscuration due to 100 pc-scale dusty structures is responsible for hiding the mirror which reflects the broad lines.

\subsection{Detectability of the polarized broad lines on the radio-FIR plane}

It is well known that a tight correlation exists between radio and far-IR (FIR) emission for normal, starburst and Seyfert galaxies (the latter with larger scatter) (Helou et al. 1985). More recently, Ji et al. (2000) have studied the radio-FIR relation of LINERs and found that the AGNand starburst-supported LINERs can be distinguished on this diagram, with the AGN-dominated ones being scattered in the region with higher radio fluxes with respect to the starburst/normal galaxies correlation. In Fig. 4 we show the radio vs. FIR diagram for the Sy2s observed in spectropolarimetry. The Sy2s with PBL are spread mostly above the standard starburst correlation (dashed line), indicating the presence of an extra contribution to the radio emission due to the AGN. Instead, Sy2s without PBL follow more tightly the starburst correlation, suggesting that in these objects, the starburst component dominates both the FIR and the radio emission.

\section{Discussion and implications}

Our finding that the absorbing column density $\left(N_{\mathrm{H}}\right)$ is marginally lower in Sy2s with PBL than in Sy2s without PBL tentatively supports the Heisler et al. (1997) model, which associates the visibility of the PBL with the amount of obscuration along the line of sight, though the statistical significance of the result is not high. Yet, the interpretation of the correlation between detectability of PBL and IR colors given by Heisler et al. is not supported by our results. They ascribe the colder IR colors observed in objects without PBL to the larger obscuration affecting the mid-IR emitting region. The mismatch between the expected and the observed IR colors in Figs. 2, 3 indicates that the IR colors are unrelated to the obscuration 
affecting the active nucleus. There are various possible scenarios (not necessarily alternative) to explain such a mismatch. For a Galactic gas-to-dust ratio and extinction curve, at $12 \mu \mathrm{m}$ the extinction implied by a column larger than $10^{23} \mathrm{~cm}^{-2}$ is higher than $2 \mathrm{mag}$, implying that, in this case, the $12 \mu \mathrm{m}$ radiation is heavily suppressed and, therefore, the observed radiation is probably dominated by the host galaxy (especially in the large IRAS beam). It is worth noting that at $N_{\mathrm{H}} \leq 10^{23} \mathrm{~cm}^{-2}$ the $25 \mu \mathrm{m} / 12 \mu \mathrm{m}$ color is lower (i.e. hotter) and follows the relation expected by the reddened Sy1 curve (dot-dashed curve in Fig. 3). Indeed, in this range of low $N_{\mathrm{H}}$, the absorbing medium is more transparent to the $12 \mu \mathrm{m}$ radiation and might well dominate over the emission from the host galaxy. At longer wavelengths $(25 \mu \mathrm{m}$ and $60 \mu \mathrm{m})$, the dust extinction is much reduced and the emitting region is much more extended. In particular, at large gaseous columns $\left(N_{\mathrm{H}}>10^{24} \mathrm{~cm}^{-2}\right)$, the medium responsible for absorption must be very compact $(<10 \mathrm{pc})$ in order not to violate constraints on the gas mass given by the dynamical mass (Risaliti et al. 1999; Maiolino 2000); as a consequence, in many cases (at least in Compton thick sources) the obscuring medium is smaller than the dusty emitting region responsible for the $25 \mu \mathrm{m}$ and $60 \mu \mathrm{m}$ radiation $(10-100 \mathrm{pc})$. Therefore, the scatter in the $60 \mu \mathrm{m} / 25 \mu \mathrm{m}$ color probably reflects mostly variations in the relative contribution of the AGN (hotter) and starburst/galactic (colder) component to the IR radiation, as suggested by Alexander (2000), although some obscuration effect on the $25 \mu \mathrm{m}$ emission might be present.

These findings confirm and strengthen the result obtained by Alexander (2000) that the relation between visibility of PBL and IR colors is mostly due to the relative dominance of AGN and the starburst/galactic component; in the sense that the latter both makes the IR colors cooler and dilutes the optical light, making more difficult the detection of the PBL. This scenario is further supported by the finding that Sy2s without PBL follow the same radioFIR correlation as starbursts (Sect. 3.4).

Yet, we find that although the relation between PBL detectability and IR colors is mostly related to the relative contribution of the starburst/galactic component, the detectability of the PBL is also affected, to a lower extent, by the obscuration toward the nuclear region. This is indicated by the larger average $N_{\mathrm{H}}$ and by the higher incidence of nuclear dusty features in Sy2s without PBL. Given the limited statistical significance of these findings ( $\sim 90 \%$ for the difference in $N_{\mathrm{H}}$ distribution), it is not surprising that such a trend was not found by Alexander (2000), who used a much smaller sample of objects.

Within the context of the relation between detectability of PBL and dominance of the starburst component, there is a possible explanation, alternative to the dilution of the optical light by the host galaxy suggested by Alexander (2000), and which might apply to some of the objects without PBL. Some authors have suggested the existence of a dichotomy in Sy2s where, at variance with the commonly accepted unified scenario, a fraction of the

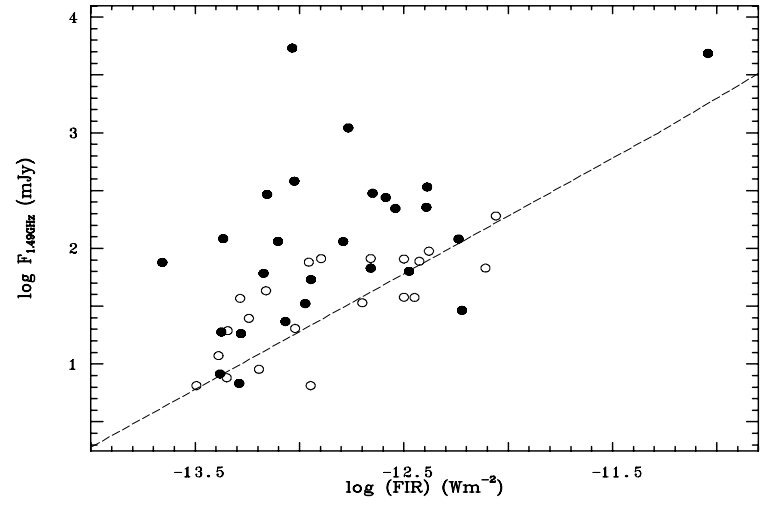

Fig. 4. Distribution of FIR and radio fluxes for the Sy2s observed in spectropolarimetry. Symbols have the same meaning as in Fig. 2. and the dashed line is the fit for normal and starburst galaxies from Helou et al. (1985)

Sy2s do not host a hidden Sy1 nucleus but are intrinsically different, such objects would be "pure" Seyfert 2 galaxies (Hutchings \& Neff 1991; Neff \& Hutchings 1992; Heckman et al. 1995; Dultzin-Hacyan et al. 1999; Gu et al. 2000). In particular the finding that the bolometric luminosity of some objects with a Sy2 spectrum is dominated by a nuclear starburst prompted Heckman et al. (1995, 1997) to argue that some extreme starburst population might mimic the narrow line spectrum of AGNs (partly supporting the model of Terlevich et al. 1992). Within this scenario, the relation between detectability of PBL and IR colors is trivial: some of the Sy2s with starburst-like cooler IR colors do not show evidence for PBL because the broad line region is absent.

\section{Conclusions}

In this paper, we collect 40 Seyfert 2 galaxies having both spectropolarimetric observations and a measure of the absorbing $N_{\mathrm{H}}$ obtained by means of their X-ray spectrum. Out of these 40 objects, 22 show broad lines in polarization (most likely ascribed to scattering of the broad line region) and 18 do not. For these objects we also analyzed the relation of $N_{\mathrm{H}}$ and detectability of the broad lines with the mid- and far-IR colors. We confirm previous claims that Sy2s without polarized broad lines have cooler IR colors. We studied additional diagrams to test two scenarios proposed to explain such a correlation and that, more specifically, ascribe this effect either to obscuration of the nuclear region or to contribution/dilution from circumnuclear starburst activity and from the host galaxy.

We found marginal evidence for Sy2s showing broad lines in polarization having lower $N_{\mathrm{H}}$. We also find that about $30 \%$ of the Sy2s without polarized broad lines show dust lanes crossing the nucleus, while none of the Sy2s with polarized broad lines show evidence for such nuclear dusty structures. These results suggest that the obscuration towards the nuclear regions (hence towards the 
scattering mirror) plays a role in hiding the polarized broad lines, at least in some objects.

On the other hand, we find that the absorbing column density does not correlate with the IR colors and, in particular, these quantities do not follow the relation expected in the case of absorption of the IR-emitting region by the dust associated to the observed $N_{\mathrm{H}}$, therefore indicating that the distribution of IR colors cannot be ascribed to obscuration effects. Also, we find that Sy2s without detection of polarized broad lines follow the same radio-FIR relation as normal and starburst galaxies, at variance with Sy2s with polarized broad lines, which tend to spread towards higher radio luminosities. These findings support previous claims that the relation between far-IR colors and detectability of the polarized broad lines in Sy2s is mostly related to dilution of the IR and optical light by a circumnuclear starburst or by the host galaxy.

Summarizing, our results indicate that the lack of broad lines in the polarized spectrum of Sy2s is mostly due to the contribution/dilution from the stellar component, though at a lower extent, the obscuration towards the nuclear region also plays a role.

Acknowledgements. We would like to thank the anonymous referee for his/her careful reading of the manuscript and valuable comments, which improved the paper very much. A significant fraction of this work was done during the Gullermo Haro Workshop 2000, we are grateful to the organizers of the workshop who made possible this collaboration and enabled us to perform this research. QSGU acknowledges support from UNAM postdoctoral program (Mexico) and from National Natural Science Fundation of China and the National Major Project for Basic Research of the State Scientific Commission of China. RM acknowledges partial support by the Italian Space Agency (ASI) under grant ARS-99-15 and by the Italian Ministry for University and Research (MURST) under grant Cofin98-02-32. And DD-H acknowledges support from grant IN 115599 from PAPIIT-UNAM. This research has made use of NASA's Astrophysics Data System Abstract Service and the NASA/IPAC Extragalactic Database (NED) which is operated by the Jet Propulsion Laboratory, California Institute of Technology, under contract with the National Aeronautics and Space Administration.

\section{References}

Alexander, D. M., Hough, J. H., Young, S., et al. 1999, MNRAS, 303, L17

Alexander, D. M. 2000, MNRAS, in press [astro-ph/0010188]

Antonucci, R., \& Miller, J. S. 1985, ApJ, 297, 621

Antonucci, R. 1993, ARA\&A, 31, 473

Awaki, H., Koyama, K., Inoue, H., \& Halpern, J. P. 1991, PASJ, 43, 195

Bassani, L., Dadina, M., Maiolino, R., et al. 1999, ApJS, 121, 473
Bohlin, R. C., Savage, B. D., \& Drake, J. F. 1978, ApJ, 224, 132

Condon, J. J., Cotton, W. D., Greisen, E. W., et al. 1998, AJ, 115,1693

Dultzin-Hacyan, D., Krongold, Y., Fuentes-Guridi, I., \& Marziani, P. 1999, ApJ, 513, L111

Granato, G. L., Danese, L., \& Franceschini, A. 1997, ApJ, 486, 147

Gu, Q. S., Dultzin-Hacyan, D., \& de Diego, J. A. 2000, submitted

Heckman, T. M., Krolik, J., Meurer, G., et al. 1995, ApJ, 452, 549

Heckman, T. M., Gonzalez-Delgado, R. M., Leitherer, C., et al. 1997, ApJ, 482, 114

Heisler, C. A., Lumsden, S. L., \& Bailey, J. A. 1997, Nature, 385,700

Helou, G., Soifer, B. T., \& Rowan-Robinson, M. 1985, ApJ, 298, L7

Hines, D. C., \& Wills, B. J. 1993, ApJ, 415, 82

Hutchings, J. B., \& Neff, S. G. 1991, AJ, 101, 434

Inglis, M. D., Brindle, C., Hough, J. H., Young, S., et al. 1993, MNRAS, 263, 895

Isobe, T., Feigelson, E. D., \& Nelson, P. I. 1986 ApJ, 306, 490

Ji, L., Chen, Y., Huang, J. H., Gu, Q. S. \& Lei, S. J. 2000, A\&Ap, 355, 922

Kay, L., Moran, E. C., Filippenko, A. V., Barth, A. J. \& Magalhaes, A. M. 2000, AAS, 196, 5013

Koyama, K., Inoue, H., Tanaka, Y., et al. 1989, PASJ, 41, 731

Maiolino, R., Salvati, M., Bassani, L., et al. 1998, A\&A, 338, 781

Maiolino, R. 2000, in X-ray astronomy '999, ed. G. Malaguti, G. G. C. Palumbo, \& N. White, in press [astro-ph/0007473]

Malkan, M. A., Gorjian, V., \& Tam, R. 1998, ApJS, 117, 25

Matt, G. 2000, A\&Ap, 355, L31

Miller, J. S., \& Goodrich, R. W. 1990, ApJ, 355, 456

Moran, E. C., Barth, A. J., Kay, L. E., \& Filippenko, A. V. 2000, ApJ, 540, L73

Moshir, M., Kopman, G., \& Conrow, T. A. O. 1992, IRAS Faint Source Survey, Explanatory supplement version 2, JPL D10015 8/92, JPL, Pasadena

Nagao, T., Taniguchi, Y., \& Murayama, T. 2000, AJ, 119, 2605

Neff, S. G., \& Hutchings, J. B. 1992, AJ, 103, 1746

Risaliti, G., Maiolino, R. \& Salvati, M. 1999, ApJ, 522, 157

Rix, H., Rieke, G., Rieke, M., \& Carleton, N. P. 1990, ApJ, 363,480

Ruiz, M., Rieke, G. H., \& Schmidt, G. D. 1994, ApJ, 423, 608

Terlevich, R. T., Tenorio-Tagle, G., Franco, J., \& Melnick, J. 1992, MNRAS, 255, 713

Tran, H. D., Miller, J. S., \& Kay, L. E. 1992, ApJ, 397, 452

Tran, H. D. 1995, ApJ, 440, 565

Veilleux, S., Goodrich, R. W., \& Hill, G. J. 1997, ApJ, 477, 631

Véron-Cetty, M. P., \& Véron, P. 2000a, A\&AR, 10, 81

Véron-Cetty, M. P., \& Véron, P. 2000b, A Catalogue of Quasars and Active Nuclei (9th edition), ESO Scientific Report, in press 